\title{
Microevolution of Candida albicans Isolate from a Patient with Mucocutaneous Candidiasis and HIV Infection
}

\author{
Gabriel Palma Cortés ${ }^{1}$, Carlos Cabello Gutierrez¹, Misael González Ibarra², \\ Magdalena Aguirre García ${ }^{3}$, Fernando Hernández Sánchez ${ }^{1}$, Haydee Torres Guerrero ${ }^{3 *}$
}

${ }^{1}$ Departamento de Investigación en Virología y Micología, Instituto Nacional de Enfermedades Respiratoria "Ismael Cosío Villegas", Ciudad de México, México

${ }^{2}$ Laboratorio de Inmuno Alergología y Micología Médica, División de Investigación, Hospital Juárez de México, Ciudad de México, México

${ }^{3}$ Departamento de Medicina Experimental, Facultad de Medicina, Universidad Nacional Autónoma de México, Hospital General de México “Dr. Eduardo Liceaga”, Ciudad de México, México

Email: ^haydeet@unam.mx, ^htorresguerrero@yahoo.com

How to cite this paper: Palma Cortés, G., Gutierrez, C.C., Ibarra, M.G., García, M.A., Sánchez, F.H. and Guerrero, H.T. (2017) Microevolution of Candida albicans Isolate from a Patient with Mucocutaneous Candidiasis and HIV Infection. Open Journal of Medical Microbiology, 7, 41-49.

https://doi.org/10.4236/ojmm.2017.72004

Received: March 7, 2017

Accepted: June 17, 2017

Published: June 20, 2017

Copyright $\odot 2017$ by authors and Scientific Research Publishing Inc. This work is licensed under the Creative Commons Attribution International License (CC BY 4.0).

http://creativecommons.org/licenses/by/4.0/

(c) $\underset{\mathrm{EY}}{\mathrm{i}}$ Open Access

\begin{abstract}
Candidiasis is the most common opportunistic fungal infection in HIV patients, and its presence is ascribed mainly to the persistence of the original infecting strain. The latter might acquire genetic variations during interaction with the host, reflecting the adaptation of the strain. Here, we report the case of a 32-year-old man complaining of asthenia, irregular hyperpyrexia, and dry cough, who was admitted to the emergency unit. Laboratory examination showed positivity for HIV. Dark violet macular lesions and ulcerated lesions with verrucous erosion were observed at the tip of the nose, whereas an ulcer without exudates was noted in the pubic region. Candida albicans was recovered from the skin by scraping these lesions. Cultures from the bronchoalveolar lavage (BAL) were negative for bacteria and opportunistic fungi but were positive for Candida albicans. The isolates from the skin and BAL were typed by PCR-RFLP and Candida albicans was identified. Analysis by microsatellite length polymorphisms, established that the pubic isolate was a genetic variant of the isolate from the nose and mouth. This suggested a microevolutionary event. Despite clinical support, the patient died of multiple organ failure.
\end{abstract}

\section{Keywords}

Candida albicans, HIV, PCR-RFLP, Microsatellite Genotyping, Microevolution 


\section{Introduction}

Candida albicans is a common commensal coloniser of the human gastrointestinal, respiratory, and reproductive tracts, as well as an opportunistic pathogen, responsible for superficial and systemic infections [1]. Mucosal Candida infections are common in patients infected with HIV. The infection is thought to result from invasion by endogenous colonising Candida in the patient; however, nosocomial outbreaks of Candida infections support the exogenous acquisition of Candida spp. as an important cause of morbidity and mortality in hospitalised patients [2]. Candidiasis is caused mainly by $C$. albicans, but other species such as Candida glabrata, Candida krusei, Candida tropicalis, Candida parapsilosis, and Candida dubliniensis have also been isolated singly and at low frequency $(1.6 \%-6 \%)$ in combination with $C$. albicans [3]. Therefore, the proper identification of the Candida species is important for choosing the appropriate therapy, as different Candida spp. display different antifungal drug susceptibilities [3] [4]. Biochemical identification of Candida spp. has been standardised using different kits; however, these approaches are often limited by variations in the phenotypic expression of different Candida species. Alternative molecular methods have been developed, which are mostly based on the amplification of specific regions, such as the 5.8S-ITS region, followed by restriction analysis [5].

Another important aspect is to determine whether the source of colonisation is a result of the selective proliferation of a single variant strain present in the commensal population before invasive infection, or a nosocomial strain acquired by cross-contamination within a hospital [6]. Patients with recurrent infections might be infected by the same strain throughout the infection period, and this strain may undergo a process of microevolution or it might be replaced by other strains [7].

The amplification of species-specific microsatellites by PCR has been successfully applied in molecular epidemiology and population studies to differentiate between $C$. albicans strains [8] [9]. The microsatellite loci CAI (CAA repeats), CAIII (GAA repeats), and CAVI (TAAA repeats) present in the $C$. albicans genome have been used for genotyping clinical isolates. This approach facilitates the differentiation of diverse isolates, as well as their epidemiological pathways. High similarity among samples suggests adaptation to environmental conditions and the occurrence of microevolutionary processes [10]. Herein, the three $C$. albicans isolates from the patient were identified using polymerase chain reaction-restriction fragment length polymorphism (PCR-RFLP). The genetic relationship among isolates was analysed by CAI, CAIII, and CAVI microsatellite genotyping. The high similarity among the three samples suggests adaptation to the environmental conditions of the isolate from the pubic region, thus confirming a microevolutionary event.

\section{Case Presentation}

We present the case of a 32-year-old HIV-infected man who was admitted to the 
emergency unit because of asthenia, irregular hyperpyrexia, and dry cough. The patient stated that he had lived in the United States for 13 years, and 4 years prior to his admission, he was diagnosed with bacterial pneumonia and treated with antibiotics. Physical examination showed acute illness, with asthenia, tachypnoea, and a small lesion suspected to be Kaposi's sarcoma. Fundoscopy revealed HIV-related retinopathy, with small necrotic spots on the inner retina and pathological dilatations of the small vessels. Dark violet macular lesions and ulcerated lesions with verrucous erosion were observed at the tip of the nose. In the pubic region, an ulcer without exudate was observed. Abundant hyaline oval yeasts, pseudomycelia, and mycelia were observed in bronchoalveolar lavage (BAL) cultures (Figure 1). The patient also developed ulcerative mucocutaneous disease due to the Herpes simplex virus, with vesicles in the eighth intercostal space of the right hemithorax. Generalised dermatosis, with vesicle-like lesions mainly on the trunk and lower limbs, was observed. Chest radiography showed diffuse bilateral interstitial infiltrates. Methenamine silver staining performed on both sputum and BAL excluded Pneumocystis jiroveci pneumonia (PJP). The initial treatment included intravenous hydration and trimethoprim/sulpha methoxazole $(20 \mathrm{mg} / \mathrm{kg} /$ day in three doses) because there was a high clinical suspicion of Pneumocystis pneumonia. Antifungal treatment with fluconazole (200 mg/kg/day) and antiviral treatment with intravenous acyclovir $(30 \mathrm{mg} / \mathrm{kg} /$ day) were started. Cultures from BAL were negative for bacteria but positive for Candida spp. Two weeks after hospital admission, the patient's overall condition continued to deteriorate despite clinical support, and he died of multiple organ failure. The aetiology of the respiratory infection could not be identified, perhaps because of the poor sensitivity of our laboratory assays.

\section{Material and Methods}

\subsection{Isolation and Identification of Candida albicans}

Three specimens, including BAL and skin lesion samples, were obtained from the patient for in vitro culture and pathogen identification via microscopic analysis. Lesion samples were taken from the tip of the nose and the pubic region using sterile swabs (Culturette, Becton, Dickinson, USA) and were processed at the Mycology Laboratory. Specimens were initially analysed by routine procedures comprising direct microscopic examination of samples with $5 \%$ $\mathrm{KOH}$ mount, and periodic-acid-Schiff and Grocott-Gomori-methenamine-silver-stained smears highly specific for $P$. jirovecii. Additional microbiological evaluations were conducted per standard procedures. Morphology testing for the presence of chlamydospores, pseudohyphae, true mycelia, and blastospore arrangement was performed on corn meal agar. The three samples were inoculated on Sabouraud Dextrose Agar (SDA) (Difco, USA) with chloramphenicol (50 $\mu \mathrm{g} / \mathrm{mL}$ ) and incubated at $28^{\circ} \mathrm{C}$ for $72 \mathrm{~h}$. For presumptive identification of Candida species, the isolates were grown on CHROMagar ${ }^{\text {ma }}$ Candida plates (CHROMagar, France). 


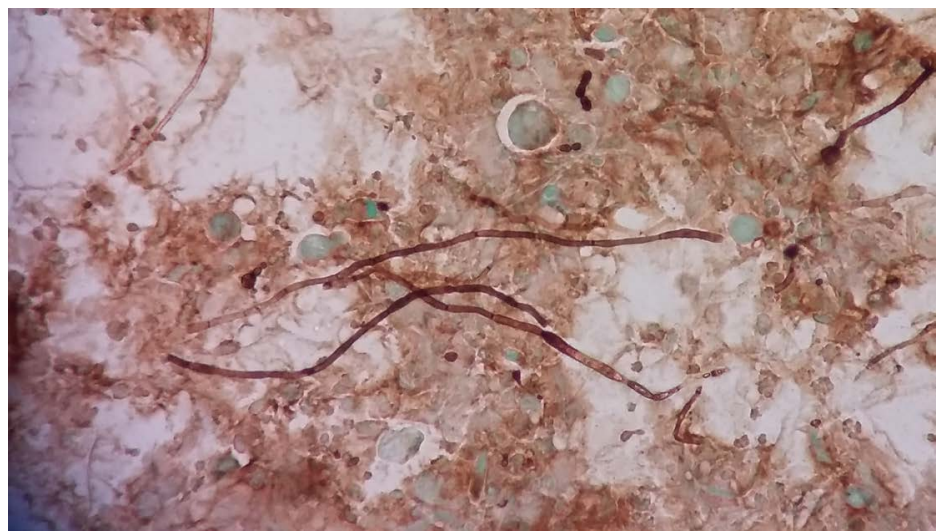

(a)

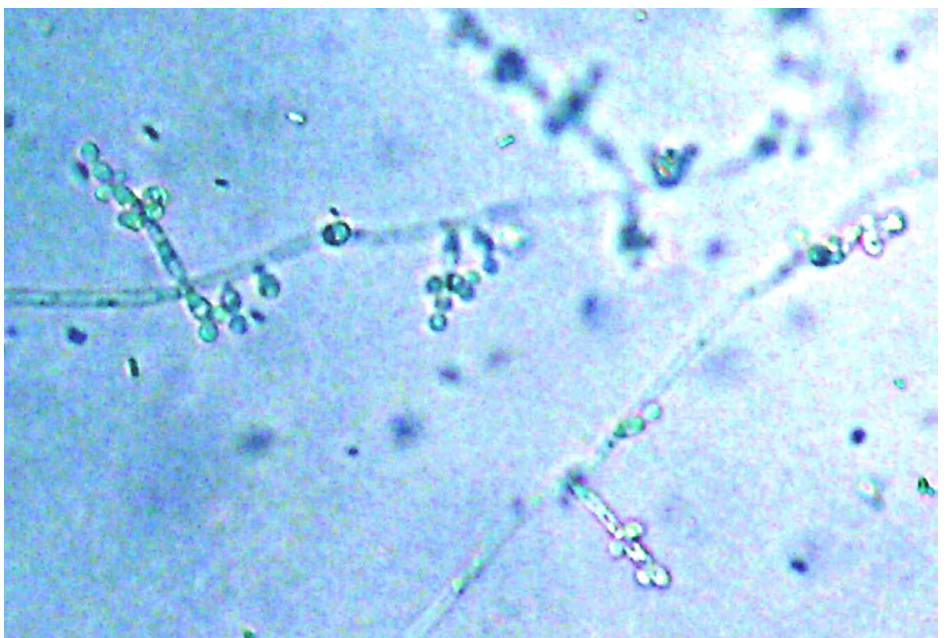

(b)

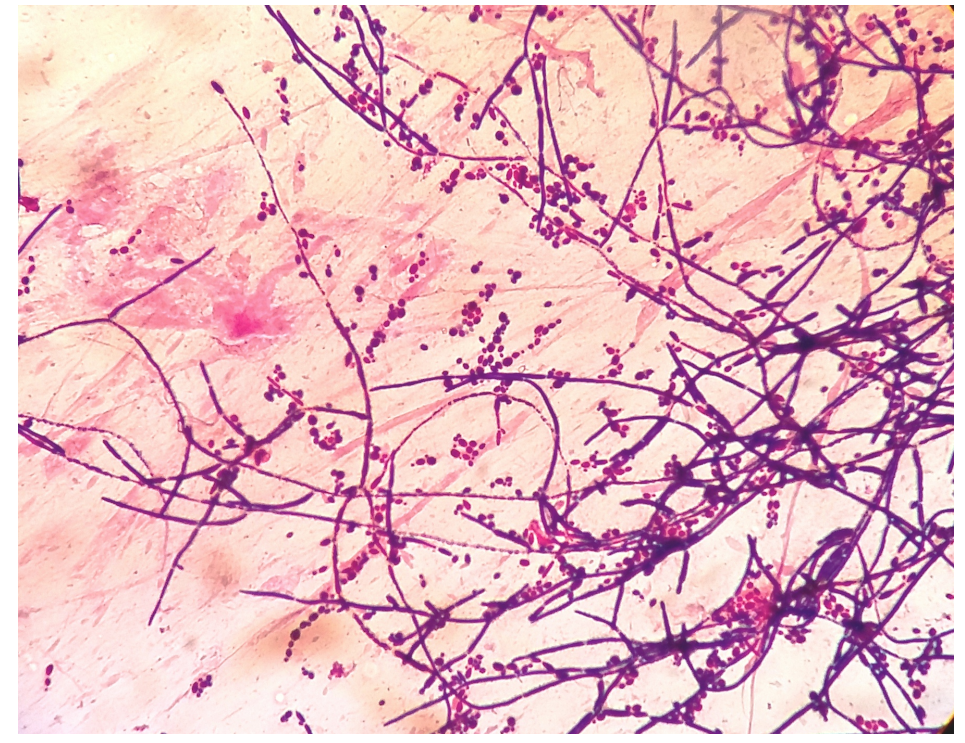

(c)

Figure 1. Cytological smears: (a) Nose skin scraping stained with Grocott's methenamine silver (GMS), hyphae, budding yeast and blastoconidia were identified; (b) Direct microscopic examination with $10 \% \mathrm{KOH}$ of pubic ulcer smear showed blasto conidia, and hyphae; (c) Periodic Acid-Schiff (PAS) stained BAL showed pseudohyphae and budding yeast. 


\subsection{DNA Purification for PCR-RFLP and Microsatellite Typing}

DNA was extracted and purified using YeaStar Genomic DNA (Zymo Research, USA). The primers used in the PCR reactions were ITS1-F (5'TCC GTAGGTGAACCTGCGG) and ITS4-R (3'TCCTCCGCTTATTGATATGC).

PCR reactions were performed in $20 \mu \mathrm{l}$ reaction mixtures containing $30 \mathrm{ng}$ of C. albicans DNA; $1 \mu \mathrm{M}$ of each primer; $0.6 \mathrm{U}$ of Taq DNA (Fermentas Inc., Canada); $200 \mu \mathrm{M}$ each of dATP, dCTP, dGTP, and dTTP; $2 \mu \mathrm{l}$ of 10xTaq polymerase buffer (Fermentas Inc.); and $2 \mathrm{mM} \mathrm{MgCl}_{2}$. The amplification products were digested with Msp1 enzyme to achieve length patterns. Restriction fragments were analysed by $2 \%$ agarose gel electrophoresis in TAE buffer; gels were stained with ethidium bromide and then photographed. C. albicans 90028, C. tropicalis 0750, C. glabrata 90030, C. krusei 6298, C. parapsilosis 22019, and Candida guillermondii 9058 were used as control strains. Genotyping with microsatellites multiplex PCR was performed in a $25-\mu \mathrm{l}$ final volume of PCR buffer (20 mM Tris HCl, pH 8.4, $50 \mathrm{mM} \mathrm{KCl),} 0.2 \mathrm{mM}$ dNTPs, $2 \mathrm{mM} \mathrm{MgCl}$, $50 \mathrm{ng}$ of genomic DNA, and 0.5 U of Taq polymerase (Applied Biosystems, USA). Forward primers used in the PCR amplification were labelled with 6-carboxyfluorescein (6-FAM) (for CAI and CAIII) and hexachlorofluorescein (HEX) (for CAVI) fluorochromes [10]. The PCR products were run on an ABI 310 Genetic Analyser (Applied Biosystems) together with the GeneScan-500 (TAMRA) size standard (Applied Biosystems). Fragment sizes were automatically determined by GeneScan 3.7 Analysis software. Alleles were designated according to their size (bp) and number of motif repeats.

\section{Results}

To identify the species of the Candida isolates, DNA was extracted from samples and control strains, after which the 5.8S-ITS region was analysed by PCR-RFLP [11]. The three isolates were identified as $C$. albicans. Antifungal susceptibility tests were performed with FUNGITEST ${ }^{\varpi}$ and results were read after incubating each isolate for $24 \mathrm{~h}$. The three Candida isolates were found to be susceptible to fluconazole $(8-64 \mu \mathrm{g} / \mathrm{mL})$, itraconazol $(0.5-4 \mu \mathrm{g} / \mathrm{mL})$, ketoconazole $(0.5-4$ $\mu \mathrm{g} / \mathrm{mL})$, myconazol $0.5-8 \mu \mathrm{g} / \mathrm{mL})$, amphotericin B $(2-8 \mu \mathrm{g} / \mathrm{mL})$ and 5-fluorocytocine $(2-32 \mu \mathrm{g} / \mathrm{mL})$.

The genetic relationship among the isolates was established with three polymorphic microsatellites: CAI, CAIII, and CAVI. Microsatellite-based genotyping was performed by multiplex PCR using automated fluorescent genotyping (Applied Biosystems \& Laragen Inc., USA) according to the protocol by Sampaio et al. [10]. For each marker and for a given isolate, one or two bands were observed. Given that $C$. albicans is diploid and each marker tested a single locus, each observed band was assigned to an allele. The genotypes observed in the patient isolates by the multiplex assay were highly related, suggesting a clonal origin. The isolates obtained from the nose and BAL were homozygous and had the same allelic combination of microsatellites as revealed by the GeneScan profiles (Figure 2). However, the genotype of the strain obtained from the pubic area 


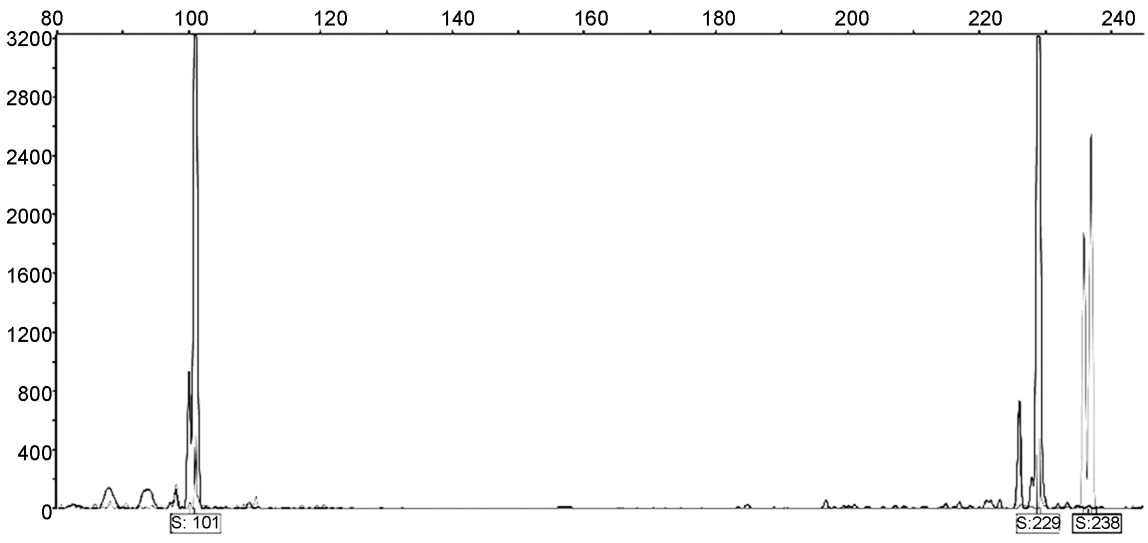

(a)

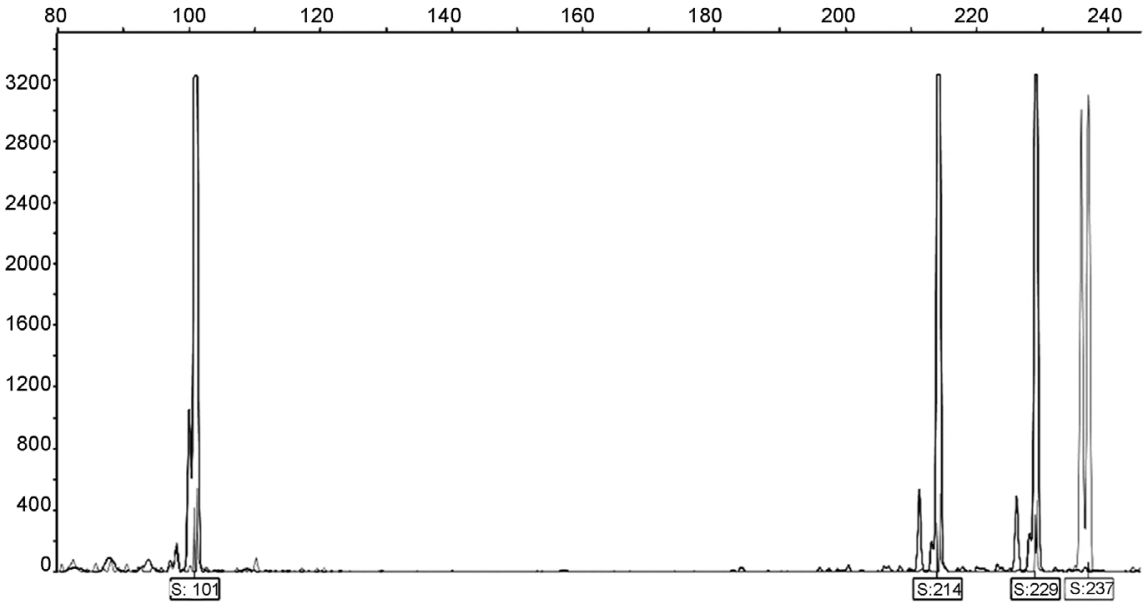

(b)

Figure 2. Gen Scan profiles representing the results of automatic fragment sizing for the microsatellite multiplex analysis for: (a) BAL and nose isolates and (b) pubic isolate (CAI) is heterozygous.

was heterozygous, suggesting that a microevolutionary event occurred at the CAI locus between genotype 38-38 and genotype 33-38 (Table 1).

\section{Discussion}

Mucocutaneous candidiasis, including oropharyngeal, oesophageal, and vulvovaginal forms, produced by $C$. albicans is the most common fungal infection in individuals with HIV [5]; however, the involvement of other Candida species has also been reported [3]. In this study, PCR-RFLP analysis of the 5.8S-ITS region clearly demonstrated $C$. albicans infection in the three analysed clinical samples. Molecular typing of these C. albicans isolates with CAI, CAII, and CAVI microsatellites revealed high similarity among the genotypes; two were identical, whereas one strain displayed a minor difference in the CAI locus. The observed allele size variation was apparently due to the deletion of five dinucleotide units (from 38 to 33). This suggests adaptation to environmental conditions and confirms a microevolution event [10]. Different studies have reported that microsatellite markers have high discriminatory power and reproducibility and 
Table 1. Alleles have been assigned according to the fragment size in bp; in parenthesis is the number of motif repeats. Genotype showing microevolution is in bold.

\begin{tabular}{ccccccc}
\hline \multicolumn{5}{c}{ Genotype of the three isolates from the patient, based on analysis of microsatellite markers } \\
\multicolumn{2}{c}{ Isolate origin } & \multicolumn{2}{c}{ CAI } & \multicolumn{2}{c}{ CAIII } & \multicolumn{2}{c}{ CAVI } \\
\hline & Alelle & Allele & Allele & Allele & Allele & Allele \\
\hline \multirow{2}{*}{1} & 2 & 1 & 2 & 1 & 2 \\
Pubis & $214(33)$ & $229(38)$ & $101(07)$ & $101(07)$ & $237(06)$ & $237(06)$ \\
Nose & $229(38)$ & $229(38)$ & $101(07)$ & $101(07)$ & $237(06)$ & $237(06)$ \\
BAL & $229(38)$ & $229(38)$ & $101(07)$ & $101(07)$ & $237(06)$ & $237(06)$ \\
\hline
\end{tabular}

are useful for studying the genetic diversity of Candida spp. [12].

Opportunistic pneumonias are the major cause of morbidity and mortality associated with HIV patients. Pulmonary complications during the evolution of the disease are mainly of infectious aetiology. The spectrum of involved pathogens is broad and includes bacteria, mycobacteria, fungi, viruses, and parasites [13]. These are responsible for opportunistic infections and malignancies, which progressively increase as $\mathrm{T} \mathrm{CD} 4^{+}$cell counts fall below 200 cells $/ \mu \mathrm{L}$. The aetiology of the pulmonary disease in the present patient could not be determined probably because of the poor sensitivity of the assays. However, C. albicans was clearly identified upon direct examination of BAL, where yeasts and mycelia were observed. The existence of Candida pneumonia as a clinical entity has been questioned. Because different species are frequently found in tracheal aspirate specimens in healthy people, the presence of Candida in sputum or other respiratory tract specimens in immunocompromised conditions is generally considered a contamination from colonisation in the upper respiratory tract. The most reliable method to confirm the clinical entity would be lung biopsy [14] [15]; however, owing to the patient's condition, this approach was not advisable.

DNA typing techniques have been extensively used for epidemiological studies in nosocomial infections and for the identification of routes of colonisation by $C$. albicans. The analysis of endogenous Candida isolates is also important because they can become invasive in immunodeficient individuals. Knowledge of the genetic relations among clinical C. albicans isolates involved in infections is important for the development and application of appropriate treatment strategies and to better understand the epidemiology of these infections.

\section{Conclusion}

Microsatellite analysis of these three isolates revealed that two of them shared the same genotype and that of the third one was also very similar. These findings suggest a common clonal origin followed by a microevolutionary event in the CAI locus.

\section{Acknowledgements}

This work was supported by grant PAPIIT-IN214013 from the Universidad Na- 
cional Autónoma de Mexico. We thank Alma Reyna EscalonaMontaño and Angelica L. Serrano Ahumada for technical assistance.

\section{References}

[1] Herwald, S.E. and Kumamoto, C.A. (2014) Candida albicans Niche Specialization: Features That Distinguish Biofilm Cells from Commensal Cells. Current Fungal Infection Reports, 8, 179-184. https://doi.org/10.1007/s12281-014-0178-X

[2] Pelroth, J., Choi, B. and Spellberg, B. (2007) Nosocomial Fungal Infections: Epidemiology, Diagnosis, and Treatment. Medical Mycology, 45, 321-346.

https://doi.org/10.1080/13693780701218689

[3] Pfaller, A.M., Andres, R.D., Diekema, J.D., Horn, L.D., Reboli, C.A., Rotstein, C., Franks, B. and Azie, N.E. (2014) Epidemiology and Outcomes of Invasive Candidiasis Due to Non-albicans Species of Candida in 2496 Patients: Data from the Prospective Antifungal Therapy (PATH) Registry 2004-2008. PLoS One, 9, 1-12. https://doi.org/10.1371/journal.pone.0101510

[4] Sanglard, D. (2016) Emerging Threats in Antifungal-Resistant Fungal Pathogens. Frontiers in Medicine (Lausanne), 15, 3-11. https://doi.org/10.3389/fmed.2016.00011

[5] Mohammadi, R., Mirhendi, H., Rezaei-Matehkolaei, A., Ghahri, M., Shidfar, M.R., Jalalizand, N. and Makimura, K. (2013) Molecular Identification and Distribution Profile of Candida Species Isolated from Iranian Patients. Medical Mycology, 51, 657-663. https://doi.org/10.3109/13693786.2013.770603

[6] Lyon, P.J., Moraes, K.C., Moreira, M.L., Aimbire, F. and Aparecida, R.M. (2010) Candida albicans. Genotyping Methods and Clade Related Phenotypic Characteristics. Brazilian Journal of Microbiology, 41, 841-849. https://doi.org/10.1590/S1517-83822010000400003

[7] Sampaio, P., Santos, M., Correia, A., Amaral, F.E., Chavéz-Galarza, J., Costa-deOliveira, S., Castro, A.G., Pedrosa, J. and Pais, C. (2010) Virulence Attenuation of Candida albicans Genetic Variants Isolated from a Patient with a Recurrent Bloodstream Infection. PLoS One, 13, No. 4, e10155.

[8] Field, D., Eggert, L., Metzgar, D., Rose, R. and Wills, C. (1996) Use of Polymorphic Short and Clustered Coding-Region Microsatellites to Distinguish Strains of Candida albicans. FEMS Immunology \& Medical Microbiology, 15, 73-79. https://doi.org/10.1111/j.1574-695X.1996.tb00056.x

[9] Sampaio, P., Gusmão, L., Alves, C., Pina-Vaz, C., Amorim A. and Pais, C. (2003) Highly Polymorphic Microsatellite for Identification of Candida albicans Strains. Clinical Microbiology, 41, 552-557. https://doi.org/10.1128/JCM.41.2.552-557.2003

[10] Sampaio, P., Gusmão, L., Correia, A., Alves, C., Rodrigues, A.G., Pina-Vaz, C., Amorim, A. and Pais, C. (2005) New Microsatellite Multiplex PCR for Candida albicans Strain Typing Reveals Microevolutionary Changes. Journal of Clinical Microbiology, 43, 3869-3876. https://doi.org/10.1128/JCM.43.8.3869-3876.2005

[11] Pires-Gonçalves, R.H., Miranda, E.T., Baeza, L.C., Matsumoto, M.T., Zaia, J.E. and Mendes-Giannini, M.J.S. (2007) Genetic Relatedness of Commensal Strains of Candida albicans Carried in the Oral Cavity of Patients' Dental Prosthesis Users in Brazil. Mycopathologia, 164, 255-263. https://doi.org/10.1007/s11046-007-9052-5

[12] Costa, C.R., de Lemos, J.A., Passos, X.S., de Araújo, C.R., Cohen, A.J., Souza, L.K. and Silva Mdo, R. (2006) Species Distribution and Antifungal Susceptibility Profile of Oral Candida Isolates from HIV-Infected Patients in the Antiretroviral Therapy Era. Mycopathologia, 162, 45-50. https://doi.org/10.1007/s11046-006-0032-y 
[13] Marcos-Zambrano, L.J., Escribano, P., Sanguinetti, M., Gómez, E., de la Pedrosa, G., De Carolis, E., Vella, A., Cantón, R., Bouza, E. and Guinea, J. (2015) Clusters of Patients with Candidaemia Due to Genotypes of Candida albicans and Candida parapsilosis. Differences in Frequency between Hospitals. Clinical Microbiology and Infection, 21, 677-683. https://doi.org/10.1016/j.cmi.2015.03.007

[14] Skalski, J.H. and Limper, A.H. (2016) Fungal, Viral, and Parasitic Pneumonias Associated with Human Immunodeficiency Virus. Seminars in Respiratory and Critical Care Medicine, 37, 257-262. https://doi.org/10.1055/s-0036-1578802

[15] Schnabel, R.M., Linssen, C.F., Guion, N., Walther, N., van Mook, C.B. and Dennis, C.B. (2014) Candida Pneumonia in Intensive Care Unit? Open Forum Infectious Diseases, 1, 1-6. https://doi.org/10.1093/ofid/ofu026

Submit or recommend next manuscript to SCIRP and we will provide best service for you:

Accepting pre-submission inquiries through Email, Facebook, LinkedIn, Twitter, etc. A wide selection of journals (inclusive of 9 subjects, more than 200 journals) Providing 24-hour high-quality service User-friendly online submission system Fair and swift peer-review system Efficient typesetting and proofreading procedure Display of the result of downloads and visits, as well as the number of cited articles Maximum dissemination of your research work

Submit your manuscript at: http://papersubmission.scirp.org/ Or contact ojmm@scirp.org 